\section{ONOMÁVEIN}

Revista semestral de lingüística, filología y traducción
PONTIFICIA UNIVERSIDAD

\title{
Los solapamientos conversacionales en edad temprana: un estudio centrado en interacciones simétricas (niño-niño) y asimétricas (niño-adulto) en español
}

Conversational Overlaps in Early Child Language:

Spanish Child-Adult and Child-Child Interactions in Focus

\section{María A. Cobelas Cartagena}

Universidad de Santiago de Compostela España

\section{(C) $($ ii) $\ominus$}

María Cobelas Cartagena: Facultade de Filoloxía, Universidade de Santiago de Compostela, España. | Correo electrónico: marian.cobelas@usc.es 


\section{Resumen}

El sistema de toma de turnos regula el intercambio de los roles de hablante y oyente entre los participantes de una conversación. Pese a que la conversación se caracteriza por un sistema de toma de turnos no marcado, existen una serie de técnicas de distribución de turnos que favorecen que el intercambio se produzca de forma fluida entre los participantes. No obstante, la sistematicidad de dicha distribución de turnos conversacionales contempla la existencia de fenómenos frecuentes, como los episodios de habla simultánea y las interrupciones que conlleva que los participantes negocien en dichos contextos ciertas estructuras de rectificación, con el propósito de garantizar la comprensión mutua durante el intercambio conversacional. El objetivo de este artículo consiste en analizar una de las transgresiones conversacionales más habituales, los solapamientos en edad temprana. Para ello, hemos llevado a cabo un estudio principalmente de corte cualitativo combinado con aspectos cuantitativos, sobre los episodios de habla simultánea producidos durante los intercambios conversacionales entre niños e iguales y entre niños y adultos. La muestra está constituida por 5 adultos y 11 niños de edades comprendidas entre los 3;0 y 4;5 años que participan en un total de 22 conversaciones en español. Nuestros resultados confirman que ya desde edad temprana los niños comienzan a gestionar las reglas que rigen el sistema de toma de turnos y utilizan el solapamiento como parte de su estrategia comunicativa.

Palabras clave: sistema de toma de turnos; solapamiento; conversaciones simétricas y asimétricas; adquisición del lenguaje infantil.

\section{Abstract}

The turn-taking system controls the exchange of roles (i.e., speaker and hearer) among the participants of a conversation. According to conversational analysts, this interactional process is governed by turn allocation techniques aimed at ensuring that this exchange takes place in a fluent, organized and systematic way. Nevertheless, there are also different conversational transgressions that break the expected rules. The aim of this study is to analyze one of the most common conversational transgressions, namely overlaps, in early child language. For this purpose, we have carried out mainly a qualitative analysis combined with some quantitative aspects focusing on the overlapping talk of Spanish child-adult and child-child conversations. We have examined a total of 22 transcripts of 5 adults and 11 children (3;0-4;5). Our results show that children are able to manage turn allocation techniques from an early age and additionally employ overlaps as a communicative strategy.

Keywords: turn-taking system; overlaps; child-adult and child-child conversations; child language acquisition. 


\section{Introducción \\ 1.1. El sistema de toma de turnos en edad temprana}

La adquisición y el desarrollo de la competencia comunicativa surgen a partir de la experiencia conversacional de la que somos partícipes los seres humanos desde los primeros días de nuestra existencia. La conversación, como defiende Tusón Valls (1997), es una de las interacciones comunicativas más habituales y espontáneas que forman parte de nuestra vida, y es a través de la cual nos convertimos en seres sociales. Por este motivo, desde principios de los 70, el interés por el estudio de la conversación y sus características surge dentro del seno de la etnometodología. Concretamente, las investigaciones de Sack, Schegloff y Jefferson (1974) son pioneras en el estudio de la organización interna de la conversación, las unidades que la componen y las reglas básicas que rigen la estructura intrínseca de la misma: el sistema de toma de turnos.

Sack y otros (1974) consideran que entre las características fundamentales de la conversación se encuentran aspectos como los siguientes: a) cambio recurrente de hablante, b) un único participante habla cada vez y c) los solapamientos (habla simultánea) son un hecho frecuente, pero de duración breve. En definitiva, lo que estos autores defienden es que el proceso de interacción se desarrolla, por norma general, de manera ordenada y sistemática. Este aspecto de la interacción se debe, según afirma Schegloff (2007), a que el sistema de toma de turno funciona de manera eficaz garantizando que, pese a la presencia de solapamientos, interrupciones o silencios, estos sean mínimos y breves. Sin embargo, trabajos como el de Gallardo-Paúls (1993) desvelan que la transición entre los turnos de una conversación está Ilena de lo que Díaz Ordaz Castillejos (2009) denomina "transgresiones a la estructura dialogal", es decir, elementos como solapamientos, interrupciones y silencios que, pese a ser previstos por el sistema, acarrean por parte de los partici- pantes la necesidad de negociar y desplegar ciertos mecanismos de rectificación, con la finalidad de solventar dichas infracciones y propiciar el intercambio de turnos de forma fluida.

Con el propósito de minimizar la existencia de dichas transgresiones conversacionales, el sistema de toma de turnos se rige por un conjunto de reglas fundamentales que permiten que los participantes de la conversación intercambien los roles de hablante y oyente de manera organizada (Sack y otros, 1974). La primera regla, la cual se aplica en el primer lugar de transición relevante (LTR), es decir, todos aquellos momentos de la conversación que pueden reconocerse a través de elementos sintácticos, prosódicos, léxicos o gestuales en los cuales se produce la alternancia de turno, se subdivide en tres variantes:

1a) En el caso de que el hablante que esté ejerciendo la palabra $(\mathrm{H} \mathrm{H})$ seleccione al próximo hablante (H2), el $\mathrm{H} 1$ debe dejar de hablar para que $\mathrm{H} 2$ pueda proceder a realizar su intervención. En la transición entre los diferentes turnos de los distintos participantes es donde tiene lugar el primer LTR, tras haber sido seleccionado el Hz. Este proceso de selección de hablante se denomina heteroselección (Sack y otros, 1974).

1b) En el caso de que un $\mathrm{H}$ no seleccione a un $\mathrm{H} 2$, cualquier otro participante tiene derecho a autoseleccionarse para iniciar el siguiente turno. Este mecanismo de selección se conoce como autoselección (Sack y otros, 1974).

1c) En el caso de que Hı no haya seleccionado a un $\mathrm{H}_{2}$ y ningún otro participante se haya autoseleccionado para llevar a cabo el siguiente turno, el Hı puede continuar ejerciendo su derecho al habla.

Por otro lado, la segunda regla consiste en la reaplicación de la regla $1(a, b, c)$ en todos los LTR subsiguientes, en el caso de que en el primer LTR no hayan operado las reglas 1 (a) y 1 (b), según la 
previsión de que el hablante que estaba ejerciendo la palabra haya continuado hablando, como postula la regla $\mathbf{I ( c )}$. Este mecanismo actúa de manera sucesiva en todos los LTR siguientes hasta que se produzca un cambio de hablante.

En otras palabras, cuando se origina un primer LTR que posibilita un cambio de turno, los hablantes pueden optar por dos opciones ante esta situación. La primera (heteroselección) consiste en indicar de forma directa al interlocutor que este está siendo seleccionado para tomar el próximo turno de habla (Sacks y Schegloff, 1973). La segunda opción (autoselección) consiste en que una de las personas que intervienen en el intercambio comunicativo, ya sea la que estaba hablando anteriormente u otro participante distinto, tome o retome su turno de palabra sin que nadie lo haya seleccionado de forma directa. Sin embargo, resulta habitual encontrar ocasiones en las que el LTR no es interpretado adecuadamente por parte del interlocutor, lo cual puede provocar la aparición de distintas transgresiones conversacionales, como, por ejemplo, el eje central de nuestra investigación: Ios solapamientos.

Ser un adulto conversacionalmente competente resulta una tarea difícil debido a que las habilidades conversacionales requieren un control de ciertos factores lingüísticos, como la planificación sintáctica y semántica de las emisiones o la comprensión y producción de las reglas pragmáticas (Acuña Robertson y Sentis Bahamondes, 2004). Por este motivo, los niños deben aprender a manejar eficientemente dos reglas fundamentales de la conversación: la cooperación y la toma de turnos, tal y como señalan Kelly Reynolds y Bailey (2013), ya que, tanto el grado de reacción por parte de los participantes en el momento adecuado como la relevancia a la hora de realizar una aportación coherente y adecuada a la línea temática que se esté desarrollando en el intercambio comunicativo resultan factores imprescindibles para participar de forma exitosa en una conversación. Las investigaciones llevadas a cabo en el ámbito del análisis de la conversación en adultos nos han permitido comprobar el complejo grupo de habilidades que los niños deben aprender desde edad temprana para poder convertirse en seres sociales comunicativamente competentes (Ninio y Snow, 1999). Sin embargo, estudios centrados en el habla infantil, como Bloom, Rocissano y Hood (1976), muestran que, a pesar de la complejidad que esto acarrea, las normas de interacción son una de las primeras pautas que los menores adquieren durante su proceso de socialización.

Existen numerosos trabajos sobre el análisis conversacional en lo que respecta al lenguaje adulto en español, entre los que destacan: Gallardo-Paúls (1993, 1996), Briz Gómez (2000), Cestero Mancera (2000) y Calsamiglia Blancafort y Tusón Valls (2002). No obstante, los estudios centrados en las habilidades conversacionales y el análisis del sistema de toma de turnos en edad temprana han sido escasos en esta lengua. Las primeras investigaciones interesadas en abordar el lenguaje infantil desde una perspectiva interaccional tienen su origen y han presentado una mayor tradición en lengua inglesa (Ervin-Tripp, 1973; Sinclair y Coulthard, 1975; Snow, 1977; Corsaro, 1979; Ervin-Tripp, 1979; Ochs y Shieffelin, 1979; Garvey, 1984; Adams y Bishop, 1989; Elias y Broerse, 1996; Clark, 2003; Berducci, 2010; Casillas-Tice, 2013). Sin embargo, en los últimos años se han publicado investigaciones en otras lenguas como, por ejemplo, el japonés Burdelski (2010), Morita (2015) o el francés Keel (2015). Dichos autores han analizado diversos aspectos que abarcan desde el estudio de la interacción infantil en etapa preverbal, las conversaciones de niños con déficit del desarrollo pragmático o la interacción en el ámbito educativo. Las distintas franjas de edad de los participantes y los diferentes ámbitos de interés que abordan estos trabajos son de carácter variado. Sin embargo, el análisis de las habilidades conversacionales en edad temprana y, concretamente, el estudio del sistema de toma de turnos y las transgresiones conversacionales en niños de entre 3;0 y 4;5 años de edad no goza 
de una larga trayectoria científica en español peninsular. Por este motivo, planteamos la presente investigación como un necesario acercamiento general al estudio de los factores implicados en la organización de la interacción infantil, especialmente, en relación al análisis de los solapamientos en conversaciones entre niños e iguales y entre adultos y niños. Nuestro trabajo defiende que la competencia comunicativa y las habilidades conversacionales son factores que se adquieren a través de la exposición del individuo al contexto social de la conversación. Dicha competencia se va adquiriendo a través de un proceso de socialización del que somos partícipes desde que nacemos hasta los últimos días de nuestra vida, coincidiendo así con autores como Keenan (1974), Bloom, Rocissano y Hood (1976), Ochs y Schieffelin (1979), Foster (1990), Ninio y Snow (1999), Blum-Kulka y Snow (2002) y Prego Vázquez (2006).

\subsection{Objetivos de la investigación}

El lenguaje infantil, como postula Tomasello (2003), presenta una serie de peculiaridades propias que no pueden ser equiparables al lenguaje adulto. Teniendo en cuenta esta premisa, el objetivo principal de este estudio consiste en desentrañar cuáles son las particularidades inherentes al lenguaje infantil en lo que respecta al manejo de la toma de turnos en edad temprana. En particular, nuestro interés está enfocado en descubrir qué ocurre y qué características presentan aquellos episodios en los que se produce un contexto de habla simultánea por parte de dos o más participantes. Con este propósito, hemos centrado nuestro estudio en la observación de cómo los niños gestionan sus participaciones dentro de la conversación. Concretamente, hemos querido averiguar si ya desde edad temprana los niños conocen y utilizan las reglas que rigen el sistema de toma de turnos. Para ello, hemos intentado dar respuesta a cuestiones como las siguientes: ¿realizan los niños en edad temprana una interpretación adecuada de los lugares de transición relevante?, ¿son frecuentes los solapamientos en las conversaciones entre niños e iguales y entre adultos y niños?, ¿qué actitud adoptan los niños cuando son solapados por sus interlocutores?, ¿qué tipos de solapamientos encontramos en este tipo de interacción?, ¿en qué momento del turno se produce la emisión de habla simultánea?, ¿existe alguna diferencia por parte de los niños cuando producen un solapamiento dependiendo de si su interlocutor es un adulto u otro niño? Responder a dichas interrogantes ha sido el propósito principal de este artículo.

\section{Método}

Hemos seguido un enfoque etnometodológico (Garfinkel, 1967) centrado en un modelo de investigación que estudia las características organizacionales del lenguaje natural en interacciones cotidianas. Siguiendo esta óptica, hemos trabajado con datos de carácter ecológico (Garner, 2004), es decir, datos reales que muestran la auténtica situación lingüística de los participantes. La metodología que se ha seguido en la realización de este trabajo refleja mayoritariamente un análisis de tipo cualitativo y descriptivo combinado con ciertas cuestiones de carácter cuantitativo. Es necesario aclarar que cuando nos referimos a los aspectos cuantitativos de nuestro análisis no aludimos en ningún caso a un análisis estadístico, sino que abordamos cuestiones numéricas como la observación frecuencial y el cálculo de porcentajes. A este respecto, hemos contabilizado una serie de elementos como: Ios turnos de habla de cada uno de los participantes (adultos y niños), los solapamientos que los niños han producido a lo largo de sus intervenciones, la frecuencia de los diferentes tipos de solapamientos encontrados y el número de veces que los niños interrumpen su aportación cuando son solapados por su interlocutor. Por otro lado, en cuan- 
to al análisis cualitativo, hemos analizado los siguientes puntos: los diversos tipos de solapamientos realizados por los menores, qué actitud tienen los niños cuando un interlocutor solapa su turno de habla, en qué lugar del turno ocurre el momento de habla simultánea y a qué interlocutor (niño o adulto) los menores solapan con mayor frecuencia.

\subsection{Materiales}

Hemos seleccionado para la realización de este trabajo un total de 22 transcripciones pertenecientes a la escuela infantil Santa Susana, localizada en Santiago de Compostela e incluida en los datos que conforman el corpus Koiné ${ }^{1}$ - elaborado por el grupo de investigación Koiné (Área de Lingüística General de la Universidad de Santiago de Compostela) - La riqueza de este corpus reside en factores como el alto número de informantes que contiene (64 individuos dentro de una franja de edad que oscila entre los 1;6 y los 4;5 años, aproximadamente) y la ecuánime distribución de variables que lo caracterizan: el género (34 niños y 30 niñas), la lengua utilizada por los informantes (español y gallego) y la diversa distribución geográfica de las distintas escuelas infantiles que lo componen (Fernández Pérez, 2011)

Para llevar a cabo el proceso de transcripción de los datos audiovisuales, se recurrió al sistema CHILDES (Child Language Data Exchange System) elaborado por MacWhinney (2000). Este importante recurso informático de almacenamiento de datos presenta numerosas ventajas, entre ellas, la posibilidad de codificar las transcripciones siguiendo el formato CHAT (Codes for the Human Analysis of Transcripts), tarea que resulta necesaria para llevar a cabo el subsiguiente rastreo y análisis de la información contenida en la nómina de transcripciones del corpus. Finalmente, para el posterior estudio de los datos se utilizó el programa de rastreo CLAN (Computerized Language Analysis). Con esta herramienta informática hemos llevado a cabo la búsqueda de los ejemplos, el recuento de los turnos y el cálculo de las frecuencias.

\subsection{Participantes}

La muestra de la presente investigación está constituida por 5 adultos y 11 niños, cuyas edades oscilan entre los 3;0 y los 4;5 años, aproximadamente. Hemos decidido centrar nuestro estudio en dicho tramo de edad debido a que autores como French y Pak (1995) y Owens (2001) consideran que alrededor de los 3;0 años es cuando los niños presentan un grado de desarrollo lingüístico verbal y manejan ciertas habilidades comunicativas que les permite adoptar un papel mucho más activo y participativo en la conversación. Por ese motivo, hemos seleccionado solo aquellas transcripciones en las que todos los participantes infantiles se encontraran en el tramo de edad que abarca desde los 3;0 años hasta los 4;5 años, edad máxima que presentan los niños que participaron en los datos recogidos en el corpus Koiné.

Las pautas que hemos considerado fundamentales para la elaboración de nuestra muestra han sido: a) la unanimidad en cuanto a la franja de edad de los sujetos (tal y como hemos explicado en el párrafo anterior), b) la pertenencia de todos los niños a la misma escuela infantil, ya que así garantizábamos que todos los sujetos se conociesen y realizaran el mismo tipo de actividades durante las grabaciones, y c) la homogeneidad en cuanto a la lengua que hablaban los menores; los 
niños de nuestra muestra utilizaban mayoritariamente el español en sus emisiones, pese a que en ocasiones muy esporádicas utilizaban también la lengua gallega². Como consecuencia de manejar dichos requisitos, los sujetos que se ajustan a los criterios previamente mencionados han sido el conjunto de los 11 niños que constituyen nuestra muestra. Este es el motivo por el cual el número de participantes infantiles analizados es un total de 11 menores y no otra cantidad distinta.

Todos los niños elegidos para este estudio presentan un desarrollo lingüístico y cognitivo normal. La paridad en cuanto al género de los participantes ha sido una cuestión que no hemos podido alcanzar, debido a las características de los datos de la muestra que hemos utilizado en este estudio. Esto se debe a que, al tomar como referente en la elaboración de nuestra muestra los criterios que acabamos de mencionar en el párrafo anterior, las 22 transcripciones derivadas de la aplicación de dichos requisitos nos ofrece únicamente un total de 10 niños y 1 niña. Por esa razón, es necesario aclarar que variables sociales, como la paridad de género en los participantes, no se han podido tener en cuenta en la realización de la presente investigación, lo cual impide la posibilidad de extrapolar los resultados derivados de nuestro estudio a cuestiones relacionadas con las posibles diferencias que pudiesen producirse en este tema según el género de los niños.

Por otro lado, pese a que el interés de nuestra investigación se basa principalmente en el análisis de las participaciones de los niños, no podemos obviar la presencia en los datos de un total de 5 adultos que desempeñan un papel fundamental en la interacción, ya que son ellos los encargados de organizar y coordinar las activida- des que se llevan a cabo durante las conversaciones grabadas. Por esta razón, nuestra muestra incluye tanto datos de conversaciones asimétricas (niños-adultos) como de conversaciones simétricas (niños-niños).

\subsection{Procedimiento}

Las grabaciones que utilizamos fueron llevadas a cabo en la escuela infantil Santa Susana (Santiago de Compostela), con una periodicidad quincenal, siempre y cuando las circunstancias lo permitían, a lo largo de varios años (1996-2000). Las investigadoras acudían a la guardería y durante un período aproximado de entre 20 y 25 minutos interactuaban de manera natural con los niños a través de una serie de actividades, como la lectura de cuentos, juegos infantiles, identificación de láminas o conversaciones espontáneas, mientras la cámara realizaba la grabación. Culminado este proceso, las conversaciones fueron transcritas y codificadas siguiendo las pautas del formato CHAT del sistema CHILDES. Por último, los datos fueron analizados con herramientas informáticas del programa CLAN, como explicaremos a continuación.

En lo que respecta al análisis de turnos y solapamientos, en primer lugar, hemos contabilizado el total del número de turnos emitidos por cada uno de los participantes, tanto de los 11 niños como de los 5 adultos, mediante la búsqueda en las transcripciones del total de emisiones encabezadas por cada uno de los nombres de los sujetos, a través del comando "KWAL" del programa CLAN. A continuación, una vez obtenido el listado de emisiones de cada participante, hemos contabilizado los turnos y comprobado cuáles y

2 Todos los niños seleccionados son de origen gallego. Por lo tanto, debemos mencionar que aunque los participantes que hemos seleccionado son niños mayoritariamente castellano hablantes, también conocen y comprenden la lengua gallega dado que el gallego es cooficial en Galicia. Sin embargo, todos los niños que componen la muestra hablan mayoritariamente español en sus intervenciones. Por lo cual, consideramos que esta investigación es relevante en lo que concierne al estudio del español de Galicia. 
cuántas de esas intervenciones eran turnos de habla simultánea. Para ello, hemos recurrido al rastreo de códigos como [<] y [>], los cuales representan en las transcripciones, respectivamente, los turnos que se encuentran seguidos o precedidos por solapamientos.

Una vez realizado el trabajo de índole más cuantitativa, hemos pasado a estudiar los aspectos de carácter cualitativo mediante la realización de un microanálisis pormenorizado de cada una de las 22 transcripciones. Por un lado, hemos observado si tales solapamientos se producían de niño a adulto o entre niños. Teniendo en cuenta este factor, hemos calculado la cantidad de solapamientos realizados en ambos casos. Por otro lado, hemos establecido una clasificación de las distintas clases de solapamientos que fueron encontrados en nuestros datos siguiendo la tipología de autores como Gallardo-Paúls (1993). Por último, hemos analizado en qué lugar del turno del hablante se produce el solapamiento y las distintas conductas de los niños en el momento en que su interlocutor solapa su turno.

\section{Resultados}

\subsection{Número de turnos y solapamientos}

Hemos contabilizado un total de 3722 turnos emitidos por los 11 niños durante las 22 conversaciones. Por otra parte, en cuanto al número total de turnos realizados por las 5 participantes adultas, hemos obtenido un resultado de 4772 turnos. Resulta esperable y comprensible que, pese al hecho de que los adultos estén en inferioridad numérica en comparación con el número de participantes infantiles en las transcripciones que analizamos, sean los adultos los que presenten una mayor participación -mayor número de intervenciones - en la conversación. Esto está motivado por el hecho de que los participantes adultos tienen como objetivo conseguir que los niños intervengan en la mayor medida posible. Por esta razón, nuestro estudio no se centra en realizar una comparativa numérica en relación a si los niños presentan mayor o menor número de solapamientos que los adultos, sino más bien lo que hemos llevado a cabo ha sido un análisis centrado en los solapamientos realizados por los niños y los distintos factores implicados en dicho fenómeno, como, por ejemplo, la tipología del solapamiento, el contexto en el que ocurren, el tipo de participante al que van dirigidos, etc.

Para ello, hemos comenzado por el cálculo de la cantidad de turnos de habla simultánea que los niños realizaron durante sus intervenciones y observamos que 574 de los 3722 turnos emitidos por los menores son solapamientos. Un 15,42\% de los turnos emitidos por los niños corresponden a casos de solapamientos. A continuación, mostraremos una tabla en la que se expone de forma detallada la información individual del total de turnos y solapamientos distribuidos por participante.

\subsection{Relación entre solapamiento y tipo de interlocutor}

Uno de los objetivos que queríamos abordar era descubrir si los menores realizaban solapamientos de manera aleatoria, independientemente de si su interlocutor fuese un adulto u otro niño, o si, por lo contrario, los solapamientos eran más habituales cuando el niño interactuaba con otro menor o con un adulto. Acercándonos a los datos podemos apreciar indicios de que los niños no solapan a sus interlocutores de manera fortuita. Las transcripciones revelan que, cuando nos encontramos ante interacciones en las que participan adultos y más de un niño, los solapamientos realizados por los menores son más frecuentes entre iguales (de niño a niño) que de niño a adulto. Concretamente, 205 de los 574 solapamientos son realizados por niños a adultos, mientras que los 369 solapamientos restantes se producen entre iguales. El 35,71\% de los solapamientos realizados por los menores a lo largo de las 22 conversaciones son solapamientos de niños a adultos, 
mientras que un 64,29\% son casos en los que un niño solapa a otro menor. En la tabla 2 podemos observar la información relativa a cada uno de los informantes.

\section{TABLA 1}

Número total de turnos y solapamientos emitidos por los 11 niños en las 22 conversaciones

\begin{tabular}{cccc} 
Informantes & $\mathbf{N}^{\circ}$ total de turnos & $\begin{array}{c}\mathbf{N}^{0} \text { de } \\
\text { solapamientos }\end{array}$ & $\begin{array}{c}\mathbf{N}^{0} \text { de turnos sin } \\
\text { solapamiento }\end{array}$ \\
\hline GUI & 201 & 6 & 195 \\
\hline DRI & 460 & 65 & 395 \\
\hline DAV & 635 & 94 & 541 \\
\hline SAN & 170 & 5 & 165 \\
\hline IEL & 23 & 1 & 22 \\
\hline TIT & 320 & 29 & 291 \\
\hline MRT & 811 & 172 & 6019 \\
\hline VIC & 718 & 117 & 16 \\
\hline CIA & 29 & 13 & 24 \\
\hline BOR & 26 & 2 & 259 \\
\hline DVD & 329 & 70 & $\mathbf{3 1 4 8}$ \\
\hline Total & $\mathbf{3 7 2 2}$ & $\mathbf{5 7 4}$ &
\end{tabular}

\section{TABLA 2}

Número de solapamientos realizados por los niños en relación al tipo de interlocutor que resulta solapado

\begin{tabular}{cccc} 
Informantes & $\begin{array}{c}\text { Solapamientos a } \\
\text { adultos }\end{array}$ & $\begin{array}{c}\text { Solapamientos a } \\
\text { niños }\end{array}$ & $\begin{array}{c}\mathbf{N}^{\circ} \text { total de } \\
\text { solapamientos }\end{array}$ \\
\hline GUI & 2 & 4 & 6 \\
\hline DRI & 31 & 48 & 65 \\
\hline DAV & 0 & 63 & 94 \\
\hline SAN & 0 & 5 & 5 \\
\hline IEL & 12 & 1 & 29 \\
\hline TIT & 70 & 17 & 172 \\
\hline MRT & 38 & 102 & 117 \\
\hline VIC & 4 & 79 & 2 \\
\hline CIA & 1 & 9 & 70 \\
\hline BOR & 30 & 1 & $\mathbf{5 7 4}$ \\
\hline DVD & $\mathbf{2 0 5}$ & $\mathbf{3 6 9}$ & \\
\hline Total & & & 13 \\
\hline
\end{tabular}




\subsection{Tipos de solapamiento}

Otro de los aspectos que hemos querido abarcar ha sido el análisis de los distintos tipos de solapamientos que hemos observado en nuestros datos. Tras el análisis, hemos hallado fundamentalmente dos variantes: solapamientos de tipo colaborativo y solapamientos de carácter competitivo. Cabe señalar que la aparición de solapamientos de índole colaborativa es casi inexistente. De hecho, su porcentaje de aparición no alcanza el 1\% del total de los ejemplos encontrados. Solo 2 solapamientos de 574 son colaborativos. Por lo tanto, los niños de entre 3;0 y 4;5 años que componen nuestra muestra emiten fundamentalmente solapamientos de tipo competitivo. A continuación, proporcionaremos un ejemplo de solapamiento colaborativo (tabla 3) y otro de carácter competitivo (tabla 4), los cuales serán comentados detalladamente en el apartado de discusión.

\subsection{Comportamiento de los niños cuan- do son solapados por sus interlocutores}

Ante una situación de habla simultánea los hablantes de una interacción pueden optar principalmente por dos opciones. La primera, y la más habitual según Hayashi (2013), es interrumpir su turno y ceder el turno de habla al interlocutor

\section{TABLA 3}

Ejemplo solapamiento colaborativo (Transcripción Sus. 5_7)

Participantes: PIL (adulta) y MRT (niño) Edad:3;00.03

*PIL: cumpleaños 〈feliz> [>] .

*MRT: <feliz> [<].

*PIL: bien!

\section{TABLA 4}

Ejemplo solapamiento competitivo (Transcripción Sus. 4_07)

Participantes: ANC (adulta), TIT (niño, edad: 3;09.27), GUI (niño, edad:4;01.05), DAV(niño, edad:3;08.03 ), DRI (niño, edad:3;10.04 )

*ANC: vale (.) y ahora la última ya (.) vamos a ver los animales.

*TIT: y yo quiero un perro : .

*GUl: y yo [/] <y yo pan con chocolate> [>] .

*TIT: <quiero un perro> [ $<]$ :

*TIT: y yo quiero (.) <un perro> [>] : .

*DAV: <chorizo> $[<]$.

*TIT: y yo quiero un perro: .

*DAV: yo quiero chorizo.

*TIT: y yo quiero (.) <un perro> [>] .

*DRI: <a mí no me gusta el chorizo〉 [<]

*ANC: a ver. 
que le ha solapado. La segunda opción consiste en que el hablante siga con su turno de habla hasta el final de su emisión, sin interrumpirla, a pesar de ser solapado por su interlocutor (Schegloff, 2000). En lo que respecta a qué actitud muestran los niños de edad temprana ante esta situación, hemos observado que solo en escasas ocasiones (un total de 5 ejemplos de los 574 solapamientos) los niños ceden su turno e interrumpen su emisión al ser solapados, lo cual contradice lo defendido por Hayashi (2013) como la opción más común que se produce en lenguaje adulto ante este supuesto. Por lo tanto, percibimos que el comportamiento mayoritario de los menores es continuar emitiendo su turno hasta completar su intervención cuando se produce habla simultánea entre los participantes. En las siguientes tablas (tabla 5 y 6) mostraremos algunos ejemplos pertenecientes a las transcripciones Sus. 4_10 y Sus. 5_8, respectivamente, de casos en los que los niños interrumpen su turno cuando son solapados.

\section{TABLA 5}

Cese del turno del hablante ante una situación de solapamiento por parte de su interlocutor

Participantes: PIL (adulta) y TIT (niño) Edad: 3;10.10

*PIL: a ver (.) tú fuche (.) tú fuche ó médico?

*TIT: 〈tú te> [>] : +//.

*PIL: 〈dixéchesme que foras ó médico〉 [<]?

*TIT: tú te Ilamas Pili.

\section{TABLA 6}

Cese del turno del hablante ante una situación de solapamiento por parte de su interlocutor

Participantes: ANI (adulta), VIC (niño, edad:3;01.05), MRT (niño, edad:3;00.17)

*ANI: ah (.) y la bruja mala (.) eh?

*VIC: sí (..) <y le trae una manzana <pero que se muere> [*] (.) y> [>] +//.

*MRT: $\left\langle\right.$ pero $\left\langle\right.$ a ti $>[/]$ a ti te llevan laz [*] brujaz $\left.\left[{ }^{*}\right]\right\rangle[<]$

\subsection{Solapamiento y lugar de transición relevante}

Averiguar el momento exacto de la conversación en el que se producen los solapamientos en interacciones asimétricas nos lleva a descubrir si los niños en edad temprana dominan o no la adecuada interpretación de los LTR que se producen entre el intercambio de turnos.

Los datos revelan que una suma de 374 solapamientos de los 574 totales tiene lugar a través de la autoselección simultánea de dos hablantes en un mismo LTR, es decir, se producen desde el inicio del turno. Este total equivale a un 65,15\% de los solapamientos realizados por los menores. Por otra parte, 200 solapamientos son iniciados a mitad del turno del primer hablante. En otras palabras, un $34,85 \%$ de los solapamientos ocurren sin esperar la llegada del LTR a la hora de realizar la intervención correspondiente por parte del segundo hablante.

Además, hemos querido comprobar si existía alguna diferencia entre ambas formas de iniciar un solapamiento en relación a quién es el participante solapado (adulto o niño). Tras estudiar los datos, descubrimos que sí existe diversidad 
en cuanto al número de solapamientos producidos dependiendo del tipo de hablante (adulto o niño) que es solapado por alguno de los menores. Por una parte, en lo que concierne a solapamientos que se producen tras la autoselección simultánea de dos hablantes en un mismo LTR, las transcripciones demuestran que un $77,56 \%$ de esta clase de solapamientos ocurre entre niños, mientras que un 22,44\% de ellos tiene lugar entre niños y adultos. Por otra parte, dentro de los solapamientos que se originan a mitad del turno, sin esperar el LTR, apreciamos que ocurre lo contrario, ya que un $60,50 \%$ de estos solapamientos se da en casos de niños a adultos, y un 39,50\% del total ocurre entre niños, como podemos observar de manera más detallada en la tabla 7.

\section{TABLA 7}

Número de solapamientos en relación al tipo de interlocutor y el momento en el que se producen

\begin{tabular}{c|c|c} 
Interlocutor solapado & $\begin{array}{c}\text { Solapamientos producidos } \\
\text { por autoselección } \\
\text { simultánea en un mismo LTR }\end{array}$ & $\begin{array}{c}\text { Solapamientos producidos } \\
\text { una vez iniciado el turno del } \\
\text { hablante }\end{array}$ \\
\hline Solapamiento de niño a adulto & 84 & 121 \\
\hline Solapamiento de niño a niño & 290 & 79 \\
\hline Total & 374 & 200 \\
\hline
\end{tabular}

\section{Discusión y conclusiones}

El objetivo principal de esta investigación ha sido descubrir cuáles eran las peculiaridades propias del lenguaje infantil en lo que respecta a la gestión de la toma de turnos en edad temprana. Concretamente, nuestro interés se centró en descubrir qué ocurría y qué características presentaban aquellos episodios en los que se producen contextos de habla simultánea por parte de dos o más participantes. A continuación, procederemos a discutir nuestros resultados más relevantes y expondremos las conclusiones obtenidas.

En el apartado anterior hemos observado que, a pesar de que el número de participantes adultos (5) es mucho menor que el de niños (11), la cantidad de turnos de los adultos es significativamente mayor que la de los niños (4722 turnos de adultos frente a 3772 turnos de niños). Este factor demuestra lo que ya hemos mencionado previamente; los adultos son los que coordinan y guían la distribución de los turnos de los participantes en aquellas conversaciones de naturaleza asimétrica (Owens, 2001; Prego Vázquez, 2004), como algunas de las que constituyen nuestra muestra. No obstante, el hecho de que los menores realicen un mayor número de solapamientos cuando el participante que está hablando es otro niño, y no un adulto, podría probar que los solapamientos no se producen de manera casual ni son producto de un desconocimiento del modo en el que operan las reglas que rigen el sistema de toma de turnos, sino que ya desde edades muy tempranas los niños empiezan a conocer las normas de interacción, como postulan Owens (2001) y Clark (2003). Además, observamos que, cuando este proceso tiene lugar, los menores empiezan también a crear estrategias comunicativas propias. Podemos apreciar que, dependiendo de quién sea el interlocutor del menor, la participación y el modo en el que los niños toman los turnos de habla varían de forma significativa. Este factor nos lleva a considerar que los menores distinguen ya una jerarquía entre los distintos participantes de la conversación. Por lo tanto, a pesar de que el número de intervenciones de los adultos es mayor, los niños tienen más cuidado al solapar un turno cuando su interlocutor es un adulto. Este hecho demuestra que la adopción del turno de habla por parte de los participantes 
de la conversación resulta sensible al contexto y a la relación jerárquica que existe entre ellos (Garvey, 1984).

Hemos distinguido en nuestros datos dos categorías distintas de solapamientos: solapamientos de carácter colaborativo y solapamientos de índole competitiva. Autores como Gallardo-Paúls (1993) definen los solapamientos colaborativos como aquellos casos en los que un segundo hablante intenta terminar el turno del participante que está ejerciendo su intervención, pronunciando, a la vez, las mismas palabras que el primer hablante. Nuestros datos demuestran que esta clase de solapamiento ya se produce en el habla infantil, pero ocurre de manera muy esporádica. En el fragmento del ejemplo (3) observamos cómo el niño comparte el turno con el adulto, diciendo a la vez las mismas palabras que su interlocutor. En este caso, observamos cómo el niño completa una frase de la tradicional canción "cumpleaños feliz". El menor ha sido capaz de completar el turno de su interlocutor gracias a una correcta identificación del contexto compartido que existe entre lo que el adulto dice en el turno anterior y lo que el niño emite en el subsiguiente turno. La escasez de solapamientos colaborativos sugiere una falta de conocimiento compartido entre los participantes de la interacción (niños y adultos), ya que, como postulan autores como Hayashi (2013), para que se pueda producir un solapamiento de carácter colaborativo es imprescindible que ambos hablantes reconozcan lo que se va a decir en la interacción. Poseer dicho tipo de conocimiento compartido es el resultado de un largo proceso de socialización que nos transforma en miembros sociales comunicativamente competentes (HernándezPina, 1984).

Por otra parte, los solapamientos competitivos (Gallardo-Paúls, 1993) son aquellos en los que un segundo hablante intenta imponer lo que este está diciendo sobre el turno de un segundo participante con la intención de arrebatarle el turno de habla. Los datos estudiados revelaron que la mayoría de los solapamientos son de índole competitiva. En el fragmento del ejemplo (4) observamos cómo 3 menores hablan de manera simultánea hasta completar su intervención sin ceder en ningún momento el turno al resto de los participantes. Autores como Hayashi (2013) consideran que los solapamientos se producen como resultado de una competición. Esta premisa nos lleva a considerar que la naturaleza de los solapamientos de los hablantes infantiles en edad temprana está ligada al afán competitivo y al habitual carácter egocéntrico que caracteriza a los niños en esas edades.

Becker Bryant (2009) considera que la toma de turnos en edad temprana resulta especialmente complicada para los niños cuando se encuentran en contextos conversacionales en los que participan múltiples interlocutores. Los datos con los que hemos trabajado son fundamentalmente interacciones en las que siempre participan más de 2 hablantes. En consecuencia, hemos observado que ciertamente este puede ser un factor que dificulte el hecho de que los niños Ileven a cabo una adecuada interpretación de los LTR a la hora de iniciar sus turnos. Por esta razón, es importante señalar que el motivo de que los datos manifiesten una mayor aparición de solapamientos iniciados como resultado de la autoselección simultánea de dos hablantes en un mismo LTR sugiere que la causa de dichos solapamientos es que los niños todavía no dominan la adecuada interpretación de los mismos. Es decir, si los solapamientos fuesen el resultado de un desconocimiento total de las normas de interacción, estos serían mucho más abundantes y ocurrirían con mayor frecuencia a mitad de los turnos del primer hablante. Sin embargo, los datos reflejan que no sucede así, ya que en nuestra muestra la autoselección simultánea de dos hablantes en un mismo LTR es la forma más común en la que se producen los solapamientos, lo cual nos lleva a considerar que existe aún una cierta falta de dominio total, por parte de los menores, a la hora de interpretar cómo y cuándo se origi- 
nan dichos LTR dentro del intercambio conversacional, pero no de las reglas que rigen el sistema de toma de turnos.

En relación con este aspecto, Ilama especialmente nuestra atención las diferencias halladas en lo que respecta al momento del intercambio conversacional en el que se produce el solapamiento y el tipo de interlocutor que resulta solapado. La presente investigación revela que los solapamientos de niños a adultos son más abundantes en aquellas ocasiones en las que estos se producen a mitad del turno del hablante que ejerce su intervención, y menos frecuentes en aquellos casos que se originan a través de la aplicación múltiple de la regla z(b) (Hayashi, 2013) en un mismo LTR (desde el inicio de turno). Podemos afirmar que esta situación está vinculada al papel coordinador que tienen los adultos en las conversaciones asimétricas. Ya que, como mencionamos anteriormente, cuando el adulto ejerce su intervención mediante el lanzamiento de una pregunta, los niños anticipan su respuesta adelantando su turno sin esperar a que se produzca el próximo LTR, con la intención de ser los primeros en responder a lo que las investigadoras están preguntando, y así poder ganar la "batalla" a sus compañeros. Este factor trae como consecuencia directa la aparición de un mayor número de solapamientos de niños a adultos producidos a mitad del turno, tal y como apreciamos en los datos.

En conclusión, coincidiendo con autores como Dore (1979), Levinson (1983) y Owens (2001), consideramos que la conversación es el contexto comunicativo más determinante e inmediato al cual nos exponemos desde edad temprana, y fuente de la cual deriva todo conocimiento lingüístico que el niño adquiere. Por esta razón, el estudio de la conversación infantil en edad temprana resulta de especial interés para las investigaciones realizadas en el campo de la adquisición del lenguaje infantil. El análisis de los solapamientos no solo resulta interesante a la hora de observar cómo se organiza la distribu- ción conversacional en edad temprana, sino que nuestros resultados también pueden ser útiles para elaborar marcos de referencia que puedan servir como elemento contrastivo en el análisis de niños con patologías lingüísticas que afecten a la vertiente comunicativa y conversacional del lenguaje, por ejemplo, en el caso de niños que presentan trastornos de déficit de atención con hiperactividad. Estos sujetos presentan síntomas que pueden afectar la distribución de los turnos en la conversación, ya que, como señala Gallardo-Paúls (2009), los menores con esta clase de trastorno, especialmente los que padecen impulsividad, se caracterizan por hablar de manera excesiva, tener problemas para esperar su turno de habla e interrumpir constantemente. Atendiendo a estos motivos, resulta evidente que nuestro trabajo puede contribuir a los avances de disciplinas como la lingüística clínica. Además, no podemos obviar que un buen desarrollo de las competencias comunicativas resulta determinante en la aceptación social de los niños en edad escolar. Los niños deben enfrentarse en el colegio no solo al hecho de saber qué decir, asimismo, los menores deben conocer también cómo decirlo (Blum-Kulka y Snow, 2002). En otras palabras, y como defienden autores como Ninio y Snow (1999) y Cabell y otros (2015), las habilidades conversacionales tienen un papel central en los diversos contextos que constituyen la vida de los niños, como, por ejemplo, el ámbito educativo o social. Es por ello que nuestra investigación es relevante y aplicable a todos aquellos aspectos relacionados con el desarrollo comunicativo infantil, especialmente en lo que respecta al español peninsular, dada la escasa existencia de estudios en esta lengua dentro de dicho ámbito científico.

\section{Bibliografía citada}

Acuña Robertson, Ximena y Franklin Sentis BahaMONDES, 2004: "Desarrollo pragmático en el habla infantil”, Onomázein 10, 33-56. 
Adams, Catherine y Dorothy V. M. BISHOP, 1989: "Conversational characteristics of children with semantic-pragmatic disorder. I: Exchange structure, turntaking, repairs and cohesion", Bristish Journal of Disorders of Communication 24, 211-239.

BeCker Bryant, Judith, 2009: "Pragmatic development" en Edith L. Bavin (ed.): The Cambridge Handbook of Child Language, Cambridge: Cambridge University Press, 339-354.

BERDUCCI, Domenic, 2010: "From infants' reacting to understanding: mature communication and sociality through turn-taking and sequencing", Psychology of Language and Communication 14 (1), 3-28.

Bloom, Lois, Lorraine Rocissano y Lois Hood, 1976: "Adult-Child discourse: developmental interaction between information processing and linguistic knowledge", Cognitive Psychology 8 (4), 521-552.

BLUm-KuLKa, Shoshana y Catherine Snow (eds.), 2002: Taking to Adults: The Contribution of Multi-party Discourse to Language Acquisition, Mahwah, NJ: Lawrence Erlbaum Associates.

Briz Gómez, Antonio, 2000: "Las unidades de la conversación”, RILCE 16 (2), 225-246.

Burdelski, Matthew, 2010: "Socializing politeness routines: Action, other-orientation, and embodiment in a Japanese preschool", Journal of Pragmatics 42, 1606-1621.

Cabell, Sonia Q., Laura M. Justice, Anita S. Mcginty, Jamie Decoster y Lindsay D. Forston, 2015: "Teacher-child conversations in preschool classrooms: Contributions to children's vocabulary development", Early Childhood Research Quarterly 30, 80-92.
Calsamiglia Blancafort, Helena y Amparo Tusón Valls, 2002: Las cosas del decir: manual de análisis del discurso, Barcelona: Arial.

CaSillas-TICE, Marisa, 2013: Learning to take turns on time: Perception and production processes involved in keeping inter-speaker gaps short. Tesis doctoral, Stanford University.

Cestero Mancera, Ana, 2000: El intercambio de turnos de habla en la conversación: análisis sociolingüístico, Alcalá de Henares: Universidad de Alcalá, Servicios de Publicaciones.

Clark, Eve, 2003: First Language Acquisition, Cambridge: Cambridge University Press.

Corsaro, William, 1979: "Sociolinguistic patterns in adult-child interaction" en Elinor Ochs y Bambi Schieffelin (eds.): Developmental Pragmatics, Nueva York: Academic Press, 373-390.

Diaz Ordaz Castillejos, Elsa M., 2009: Prácticas comunicativas e identidad en el aula desde el análisis del discurso. Tesis doctoral, Universidad de Santiago de Compostela, Santiago de Compostela.

Dore, John, 1979: "Conversational acts and the acquisition of language" en Elinor Ochs y Bambi Schieffelin (eds.): Developmental Pragmatics, Nueva York: Academic Press, 339-362.

EliAs, Gordon y Jack Broerse, 1996: "Developmental changes in the incidence and likelihood of simultaneous talk during the first two years: a question of funtion", Journal of Child Language 23 (1), 201-217.

ERvin-Tripp, Susan, 1973: Language acquisition and communicative choices / Essays by Susan ErvinTripp; selected and introduced by Anwar S. Dil. Stanford: Standford University Press.

ERvin-TRIPP, Susan, 1979: "Children's verbal turntaking” en Elinor Ochs y Bambi Schieffelin (eds.): 
Developmental Pragmatics, Nueva York: Academic Press, 391- 414.

Fernández Pérez, Milagros (coord.), 2011: Lingüística de corpus y adquisición de la lengua, Madrid: Arco Libros D.L.

Foster, Susan H., 1990: The Communicative Competence of Young Children: A Modular Approach, Longman: New York.

French, Lucia, y Meesook KIM PAK, 1995: "Young children's play dialogues with mothers and peers" en Keith E. Nelson y Zita Réger (eds.): Children's Language, Erlbaum: Hillsdale, 8, 65-101.

Gallardo-Paúls, Beatriz, 1993: "La transición entre turnos conversacionales: silencios, interrupciones y solapamientos", Contextos XI (21), 189-220.

Gallardo-Paúls, 1996: Análisis conversacional y pragmática del receptor, Valencia: Episteme.

GallaRdo-PAúls, 2009: Trastorno por déficit de atención y/o hiperactividad, volumen V, Corpus PerLA, Valencia: Guada Impresiones S.L.

GaRfInKel, Harold, 1967: Studies in Ethnomethodology, Cambridge: Polity Press.

Garner, Mark, 2004: Language: an ecological view, Oxford: Peter Lang.

Garvey, Catherine, 1984 [1987]: Children's Talk. Cambridge, Mass: Harvard University Press. [Trad. cast. de Ediciones Morata, S. L, El Habla Infantil. Madrid: Ediciones Morata, S. L.]

HaYASH, Makoto, 2013: "Turn allocation and turn sharing” en Jack SIDNELL y Tanya STIVERS (eds.): The Handbook of Conversation Analysis, Malden, MA: Wiley-Blackwell, 167- 190.

Hernandez-Pina, Fuensanta, 1984: "Actitudes lingüísticas parentales y desarrollo del lenguaje infantil", Infancia y Aprendizaje 21, 35-60.
KeEL, Sara, 2015: "Young children's embodied pursuits of a response to their initial assessments", Journal of Pragmatics 75, 1-24.

Keenan, Elinor, 1974: "Conversational competence in children”, Journal of Child Language I (2), 163-183.

Kelly, Kimberly Reynolds y Alison L. Bailey, 2013: "Dual Development of Conversational and $\mathrm{Na}$ rrative Discourse: Mother and child interactions during narrative co-construction", Merrill-Palmer Quarterly 59, 4, 426-460.

Levinson, Stephen, 1983: Pragmatics, Cambridge: Cambridge University Press.

MacWhinney, Brian, 2000: The Childes Project, Hillsdale, NJ: LEA.

MorItA, Emi, 2015: “'Say [x]': A Device for Securing Conversational Footing in the Talk of Young Children”, Discourse Processes, 00; 1-21.

Ninio, Anat y Catherine Snow, 1999: "The development of pragmatics: learning to use language appropriately" en William Ritchie y Tej BhatıA (eds.): Handbook of Child Language Acquisition, San Diego: Academic Press, cop, 347-383.

Ochs, Elinor y Bambi Schieffelin (eds.), 1979: Developmental Pragmatics, Nueva York: Academic Press.

Owens, Robert, 2001 [2003]: Language development: an introduction, quinta edición, Boston: Allyn and Bacon. [Trad. cast. de A. J. Escudero, Desarrollo del Lenguaje. Madrid: Prentice Hall, D.L.]

Prego Váquez, Gabriela, 2004: "Narraciones de niños de tres y cuatro años: un puente entre la gramática y la pragmática” en Montserrat VeYRAT Rigat y Beatriz Gallardo-Paúls (eds.): Estudios de lingüística clínica: aspectos evolutivos, Valencia: Universitat de Valencia-Estudi General / AVaLCC, 99-126. 
Prego Vazquez, Gabriela, 2006: "Code-switching emergente y voces discursivas en el habla infantil" en Milka Villayandre Llamazares (ed.): Actas del XXXV Simposio Internacional de la Sociedad Española de Lingüística, León: Universidad de León, 1526-1545.

Sacks, Harvey y Emmanuel Schegloff, 1973: "Opening up closing", Semiotica 7 (4), 289-327.

Sacks, Harvey, Emmanuel Schegloff y Gail Jefferson, 1974: "A simplest systematic for the organisation of turn-taking for conversation”, Language 50 (4), 696-735.

Schegloff, Emmanuel, 2000: "Overlapping talk and the organization of turn-taking for conversation", Language in Society 29, 1-63.

Schegloff, Emmanuel, 2007: Sequence Organization in Interaction, Nueva York: Cambridge University Press.
SinCLAIR, John y Malcolm CoulthaRd, 1975: Towards an analysis of discourse: The English used by teachers and pupils, Londres: Oxford University Press.

Snow, Catherine, 1977: "The development of conversation between mothers and babies", Journal of Child Language 4, 1-22.

Tomasello, Michael, 2003: Constructing a Language. A Usage-Based Theory of Language Acquisition, Cambridge, Mass: Harvard University Press.

Tusón Valls, Amparo, 1997: Análisis de la Conversación, Barcelona: Ariel.

\section{Anexos}

\subsection{Información de las transcripciones} utilizadas

\begin{tabular}{|c|c|c|c|}
\hline $\begin{array}{l}\text { Nombre de la } \\
\text { transcripción }\end{array}$ & Fecha & $\mathrm{N}^{\circ}$ de participantes & Duración \\
\hline Sus3_01 & 21-mai-97 & 2 & $25^{\prime} 28^{\prime \prime}$ \\
\hline Sus3_02 & 04-xun-97 & 2 & $09^{\prime} 28^{\prime \prime}$ \\
\hline Sus3_03 & 11-xun-97 & 2 & $21^{\prime} 46^{\prime \prime}$ \\
\hline Sus3_04 & 25-xun-97 & 2 & $14^{\prime} 22^{\prime \prime}$ \\
\hline Sus4_04 & 04-dec-97 & 5 & $21^{\prime} 45^{\prime \prime}$ \\
\hline Sus4_05 & 15-xan-98 & 4 & $13^{\prime} 37^{\prime \prime}$ \\
\hline Sus4_06 & 03-feb-98 & 3 & $09^{\prime} 48 "$ \\
\hline Sus4_07 & 12-feb-98 & 6 & $19^{\prime} 23^{\prime \prime}$ \\
\hline Sus4_08 & 26-feb-98 & 5 & $13^{\prime} 50^{\prime \prime}$ \\
\hline Sus4_09 & $02-a b r-98$ & 5 & $15^{\prime} 07^{\prime \prime}$ \\
\hline Sus4_10 & 18-xun-98 & 4 & $15^{\prime} 41 "$ \\
\hline Sus4_11 & 17-abr-98 & 5 & $04^{\prime} 21^{\prime \prime}$ \\
\hline Sus4_12 & 30-abr-98 & 5 & $13^{\prime} 12^{\prime \prime}$ \\
\hline Sus4_13 & 14-mai-98 & 4 & 09' 09" \\
\hline Sus4_14 & 28-mai-98 & 5 & $07^{\prime} 37^{\prime \prime}$ \\
\hline Sus5_07 & 17-feb-99 & 6 & $15^{\prime} 40^{\prime \prime}$ \\
\hline Sus5_08 & 03-mar-99 & 3 & $19^{\prime} 28^{\prime \prime}$ \\
\hline
\end{tabular}




\begin{tabular}{llll}
\hline Sus5_09 & 18-mar-99 & 4 & $17^{\prime} 39^{\prime \prime}$ \\
\hline Sus5_10 & 08-abr-99 & 5 & $21^{\prime} 37^{\prime \prime}$ \\
\hline Sus6_01 & 26-abr-99 & 4 & $19^{\prime} 19^{\prime \prime}$ \\
\hline Sus6_02 & 06-mai-99 & 5 & $18^{\prime} 41^{\prime \prime}$ \\
\hline Sus6_03 & 27-mai-99 & 4 & $18^{\prime} 13^{\prime \prime}$ \\
\hline
\end{tabular}

\subsection{Datos de los informantes}

\begin{tabular}{c|c|c|c|c} 
Participantes & $\begin{array}{c}\text { Edad primera } \\
\text { grabación }\end{array}$ & $\begin{array}{c}\text { Edad última } \\
\text { grabación }\end{array}$ & $\begin{array}{c}\mathbf{N}^{\circ} \text { de } \\
\text { grabaciones }\end{array}$ & $\begin{array}{c}\text { Género de los } \\
\text { informantes }\end{array}$ \\
\hline GUI & $3 ; 04.28$ & $4 ; 04.21$ & 17 & $\mathrm{~m}$ \\
\hline DRI & $3 ; 07.26$ & $4 ; 01.20$ & 11 & $\mathrm{~m}$ \\
\hline DAV & $3 ; 00.13$ & $3 ; 02.14$ & 9 & $\mathrm{~m}$ \\
\hline SAN & $3 ; 09.17$ & $4 ; 04.01$ & 5 & $\mathrm{~m}$ \\
\hline IEL & $3 ; 08.29$ & $3 ; 08.29$ & 1 & $\mathrm{~m}$ \\
\hline TIT & $3 ; 09.18$ & $4 ; 02.02$ & 7 & $\mathrm{~m}$ \\
\hline MRT & $3 ; 00.03$ & $3 ; 03.26$ & 7 & $\mathrm{f}$ \\
\hline VIC & $3 ; 00.22$ & $3 ; 04.14$ & 8 & $\mathrm{~m}$ \\
\hline CIA & $3 ; 00.20$ & $3 ; 01.02$ & 2 & $\mathrm{~m}$ \\
\hline BOR & $3 ; 00.25$ & $3 ; 00.02$ & 1 & 5 \\
\hline DVD & $3 ; 00.13$ & $3 ; 02.14$ & 7 &
\end{tabular}

